\title{
Die Familie in Georg K. Glasers, Geheimnis und Gewalt‘ vor dem geschichtlichen Hintergrund
}

\author{
Pavel KNÁPEK
}

\begin{abstract}
The historical context of the family in Georg K. Glaser's novel Secret and Violence

The article focuses on the aspect of family in the period after the First World War as portrayed in Georg K. Glaser's novel Secret and Violence (Geheimnis und Gewalt). The novel depicts the division of a family caused by the brutality of the father, who has returned from the war and has become completely estranged from his family. The paper finds a parallel between the plot of the novel and the political and social events that unfolded in Germany during the first half of the 20th century, which split society and thus often caused a complete alienation among people. In the novel, the Weimar Republic is presented as a battleground of progressive and radically ideological forces, whose success the study seeks to elucidate.
\end{abstract}

Keywords: Georg K. Glaser; Secret and Violence; Klaus Theweleit; Male fantasies; German literature

DOI: $10.15452 /$ StudiaGermanistica.2021.29.0006

\section{Einleitung}

Die Hauptfigur in Georg K. Glasers ,Geheimnis und Gewalt' heißt Valentin Haueisen und sein Leben wird von dessen früher Kindheit bis zur Befreiung aus der nationalsozialistischen Gefangenschaft geschildert. Das Werk ist nicht die letzte Prosa des Autors, jedoch seine berühmteste. Sie kann als eine existenziell ausgerichtete Reflexion von Haueisens Leben vor dem Hintergrund der Epoche zwischen ca. 1918 und 1945 bezeichnet werden. Haueisen ist ein Alter Ego des Verfassers; allerdings handelt es sich in diesem Fall nicht um eine Autobiographie. Trotz vieler Details, die mit seinem Leben übereinstimmen, erhebt Glaser Anspruch auf ein genuin literarisches Werk, das eine EssenZ DEs Menschlichen sowie des ZeitTyPischen darstellen soll. Michael Rohrwasser (1991) erklärt die Arbeitsweise des Autors folgendermaßen: „Glasers Bemühen ist auf ein ,Wesentliches“ gerichtet; er bezeichnet seine Schreib- und Lebensarbeit als, Verdichtung', mit der das Überflüssige fortgenommen wird.“ (Rohrwasser 1991:253). Das Schaffen des Schriftstellers sieht er dennoch und mit Recht - als einen literarisch „,verdichteten“ und verfremdeten Lebensbericht, dem „die Biographie des Autors zugrunde liegt.“( (ebd.:242). Wer deutsche Geschichte in Zusammenhängen 
verstehen will, erfährt aus Glasers Prosa sehr viel. Besonders die Voraussetzungen für die Entstehung der beiden totalitären Regime in Deutschland des 20. Jahrhunderts werden aus dem Erzählten klar ersichtlich, ohne dass das Werk sich mit ihnen auf eine politisch-theoretische Weise befassen würde. Es handelt über Menschenschicksale.

Im vorliegenden Artikel werde ich die Familienkonstellation in ,Geheimnis und Gewalt ${ }^{`}$ untersuchen und parallel dazu einige Aspekte des politisch-gesellschaftlichen sowie privaten Lebens in der Weimarer Republik herausarbeiten, um zum Verständnis der Familienbeziehungen und des Romangeschehens in Glasers Hauptwerk einen Beitrag zu leisten. Zur Analyse der Figur des gewalttätigen Vaters, die im Rahmen des vierten Kapitels dieser Arbeit untersucht wird, werde ich Klaus Theweleits ,Männerphantasien“ heranziehen, bzw. diejenigen Teile dieses Werks, die auf überprüfbaren Tatsachen beruhen und die die geschichtlich bedingte Mentalität von soldatischen Männern erklären. In dieser Analyse wird die Primärliteratur in Bezug zur Geschichte gesetzt.

\section{Die Epoche von 1918 bis 1933 in Deutschland als Hintergrund der Literatur}

Die Gründung der Weimarer Republik sowie die ersten Jahre ihrer Existenz hat der Bürgerkrieg geprägt. Der regierenden SPD wurde Verrat an der Arbeiterschaft vorgeworfen, da ihr Minister die Niederschlagung des Spartakusaufstands (1919) durch die berüchtigten Freikorps anordnete, welche ebenfalls die Führer des kommunistischen Spartakusbundes Rosa Luxemburg und Karl Liebknecht ermordeten. Später beteiligten sich die Freikorps am Kapp-Putsch, dessen Ziel wiederum der Sturz der neu gebildeten Regierung war. Die Weimarer Republik war oft außerstande die elementarsten Bedürfnisse der ärmsten Sozialschichten zu gewährleisten - in erster Linie wohl infolge der enormen Reparationszahlungen. Die Arbeitsämter konnten den Arbeitslosen - auch vor der großen Weltwirtschaftskrise - in zahlreichen Fällen weder Arbeit noch Geld geben (vgl. Glaser 2007:151-157). Die ärmsten Bevölkerungsschichten litten unter Hunger. Die Arbeiter und Arbeiterinnen der Fabriken haben sich infolge der langen Arbeitszeiten und des Hetztempos der Maschinen (die Einführung des Fließbands) in vielen Fällen Verletzungen und Verstümmelungen zugezogen. In den chemischen Betrieben wurden sie - meist ohne wirksame Schutzmittel - der Wirkung hochgiftiger Stoffe ausgeliefert, so dass häufig lebenslange Gesundheitsschäden oder gar Todesfälle infolge kleinster Manipulationsfehler vorkamen. Otto Rühle (1922) macht unter anderem auf die katastrophalen Wohnverhältnisse des Proletariats (nicht nur) in Großstädten aufmerksam. Die Situation habe sich in Berlin wegen des Bauverbots während der Kriegsjahre sowie infolge des Zustroms der Flüchtlinge weiter verschlechtert (vgl. Rühle:141ff.). In den Wohnungen der niedrigeren Arbeiterklasse hätten so viele Menschen gelebt, dass Zimmer und Betten von mehreren Personen geteilt werden mussten. Rühle schreibt: „Insgesamt mussten jahraus jahrein in Berlin rund 600.000 Menschen in Wohnungen hausen, bei denen mehr als 5 Personen auf jedes Zimmer kamen.“ (ebd.:141). Die Dichte der in einem Haushalt lebenden Menschen hatte hohe Ansteckungsraten zur Folge: Die größte Gefahr war die Tuberkulose - die „Krankheit der Armen“. Das Leben des Proletariats ${ }^{1}$ in den Innenräumen der Großstädte war durch Staub, Schmutz, Lärm, Kälte, Feuchtigkeit, Schimmel und Ungeziefer gezeichnet. So beschreibt Rühle einen typischen Arbeiterhaushalt:
„Das Mobiliar, die Tapeten, die Wände und Decken sind fast überall in einem Zustande fortgeschrit- tener Verwahrlosung. = Die Wohnung, wo die Tapete von den Wänden hängt und die Feuchtigkeit bereits einen Überzug von Schimmel und von Pilzen an der Wand gebildet hat, ist keine Ausnahme; sondern im Gegenteil: diese Verwahrlosung ist die Regel.“ (ebd.:142f.)

Trotz aller Schwierigkeiten und unheilvollen Tendenzen, auf die ich im folgenden Kapitel eingehen werde, sind mit dem Begriff Weimarer Republik ebenfalls viele radikal liberale Tendenzen und ein enormer Aufschwung der Wissenschaft verknüpft. Berlin wurde weltweit zu ihrem wichtigsten

Zugunsten besserer Lesbarkeit verwende ich meistens nur die männliche Form. Frauen und andere Geschlechter sind jedoch stets mitgemeint. 
Zentrum: Seit 1915 war Albert Einstein Professor in Berlin und von 1917 bis 1933 Direktor des Berliner Kaiser-Wilhelm-Instituts für Physik. Einsteins Berufung nach Berlin hat der dortige Professor Max Planck vermittelt - beide wurden mit dem Nobelpreis für Physik ausgezeichnet. Der Sexualwissenschaftler Magnus Hirschfeld setzte sich weltweit als erster für die Entkriminalisierung der Homosexualität ein - wenn auch erfolglos. In den 1920er Jahren wurde er wegen seiner Arbeit sowie wegen seiner jüdischen Herkunft zur Zielscheibe von nationalsozialistischen Hetzkampagnen und schweren physischen Angriffen.

Ähnlich wie in anderen Ländern Europas war man in Deutschland der Zwischenkriegszeit um reformpädagogische Konzepte bestrebt, die jedoch in der Praxis von der traditionellen Prügelpädagogik in den Schatten gestellt wurden. ${ }^{2}$ Bekannt sind unter anderem die von Rudolf Steiner begründeten Waldorfschulen oder die fortschrittlich liberalen Konzepte der Leitung von Erziehungsheimen - unter ihnen das Frankfurter Westendheim August Verlegers. ${ }^{3}$ Eine gute Übersicht zum Thema Reformpädagogik liefert Michael Rohrwasser (2007) im Nachwort zu ,Schluckebier'. Zu anderen progressiven Initiativen in der Weimarer Republik gehörten vielfältige Initiativen für Tierrechte. Ihre Vertreter durchschauten dabei allzu gut die Strategie der Nationalsozialisten, denen es trotz dem proklamierten Interesse am Tierschutz darum ging, aus der jüdischen rituellen Tierschlachtung ein Instrument der Judenhetze zu machen. Der Baltendeutsche Manfred Kyber schrieb 1925 gleich auf der ersten Seite seiner Schrift ,Tierschutz und Kultur', dass sich JEDES Volk der Erde in Bezug auf die Tatsache, dass es Grausamkeiten gegen Tiere begeht, als unedel und niedrig bezeichnen müsse. Unmissverständlich - in Anspielung auf die chauvinistischen Tendenzen im damaligen Deutschland - fuhr er fort: ,Es ist auch keine patriotische Tat, sein eigenes Volk mit großem Geschrei und gewaltiger Empörung in Schutz zu nehmen und es als das allererste Kulturvolk der Welt hinzustellen. Das ist billiger Hurra-Patriotismus.“ (Kyber 1925:5f.). Manfred Kybers Werke (vor allem die ,Tiergeschichten") haben dem Autor viel Erfolg eingebracht. In Deutschland erschienen sie in der Auflage von 200.000 Exemplaren (vgl. Brieger 1973:248) und sie müssen daher einen gewissen Einfluss auf die jugendlichen Leser ausgeübt haben. ${ }^{4}$ Kybers pazifistischer Geist der Liebe und Respekts zu allen Menschen und Tieren stand freilich im scharfen Gegensatz zu viel verbreiteten Ideen des Sozialdarwinismus, Militarismus, Rassismus sowie des radikal revolutionären Kommunismus. In diesem Sinne zitiert Anton Brieger (1973) aus einer Rezension über Kybers, Tiergeschichten', die dem Autor folgende Attribute zuordnet: Wehleidigket; Albernheit; Sentimentalität; der Inhalt der Geschichten als ein schwachseliges und weichherziges Gewinsel. Im Ganzen sei das Buch das „Produkt eines dekadenten, sentimentalen oder hysterischen Tierschutzvereinlers,“ eines „kleinen, kleinlichen Geist[es]“ und für „gesund denkende Männer peinige[nd]“ (vgl. Brieger 1973:248). Der bereits kranke Manfred Kyber starb fast unmittelbar nach Hitlers Kanzleramtsantritt am 10. März 1933.

Die in diesem Kapitel angeführten Beispiele sollten die politische, wirtschaftliche und geistige Spaltung in Deutschland jener Epoche illustrieren. In Georg K. Glasers ,Geheimnis und Gewalt` lesen wir in diesem Sinne:

Bahnbrecher, Verkünder und Jünger vergangener und zukünftiger Glaubenslehren [sehnten sich nach dem Kontakt mit der Jugend. Oft waren sie liebenswürdig und ehrlich] ... Seltsam jedoch war erst, daß alle felsenfest davon überzeugt waren, den einzigen Hebel zu besitzen, der die Welt aus den An-

Nach mehreren medialisierten Skandalen in Erziehungsheimen wurden im Jahre 1929 körperliche Strafen in allen Fürsorgeanstalten verboten (vgl. Rohrwasser 2007:335).

3 Glasers Erzähler über die ursprüngliche Vision der Erziehungsanstalt „Billigheim“, deren Inspiration das von Verleger geführte Westendheim (Frankfurt am Main) darstellte:

Es war verboten, zu schlagen. Die Zöglinge arbeiteten in Werkstätten und Betrieben wie jedermann. Die Anstalt war nur Betreuung und Heim. [...] die seltsamen Gärtner beteuerten mir, daß sie für mich da waren, und sie bewiesen es: sie bemühten sich um mein leibliches Wohl, baten und ermutigten mich, Wünsche zu haben, und buchten es als Sieg, wenn ich sie äußerte. (Glaser 1989:6).

4 „Fremdsprachige Übersetzungen, so ins Finnische, Schwedische, Englische, Tschechische und Holländische, lagen schon vor dem Zweiten Weltkrieg vor, und die Aufnahme einzelner Erzählungen in amerikanische Schulbücher bewiesen schon damals das lebhafte Interesse des Auslandes am Werk des Dichters.“(vgl. Brieger 1973:248). 
geln heben konnte. ... Das Leben war bis in seine winzigsten Ausdrücke, bis in die Schlafstuben, bis in jedes Wort durchtränkt von dem Zwang der Wahl. (ebd.:38-40).

Der Bürgerkrieg, der zumindest die Anfänge der Weimarer Republik begleitete, drohte in einen Klassenkrieg zu münden. Am Ende war - laut der Doktrin der 1933 siegreichen Nationalsozialisten - das Weltjudentum an der Niederlage Deutschlands für schuldig befunden. Seine entscheidende Wirkung vermuteten sie sowohl im internationalen Bolschewismus als auch im globalen Großkapitalismus - allesamt Kräften, die es - der NS-Propaganda zufolge - auf vollständige Vernichtung Deutschlands abgesehen gehabt hätten.

\section{Das Erbe des Wilhelminismus}

In seinem viel beachteten Buch ,Männerphantasien“ (1978; Neuauflage 2019) versucht der Kultur- und Literaturhistoriker Klaus Theweleit die Voraussetzungen des deutschen Faschismus ${ }^{5}$ zu skizzieren, indem er verschiedene Schriften der (oder über die) Freikorpssoldaten, die sowohl gegen die kommunistischen Aufständischen (mit Billigung der Regierung), die russische Rote Armee im Baltikum, die polnischen Aufständischen in Oberschlesien als auch am Ende gegen die Regierung der Weimarer Republik selbst kämpften. Der Autor untersucht Schriftwerke (Romane, Briefe, Autobiographien, Erinnerungsschriften) von 7 als (unmittelbar nach dem Ersten Weltkrieg) faschistisch zu bezeichnenden Männern. Neben den Kommentaren und Ausführungen des Verfassers beinhaltet das 1118-seitige Werk lange Zitate aus den Schriften nicht nur jener erwähnten Protagonisten, sondern soldatisch antirepublikanisch eingestellter Männer überhaupt. Die Zitate vermitteln den Lesern ein äußerst verstörendes Bild. Theweleit weist vor allem auf den Hass der Freikorpsmitglieder gegen proletarische Frauen, die als „Huren“ und hämische „Flintenweiber“ dämonisiert und hemmungslos getötet werden. Die im Buch präsentierten Texte dokumentieren aber auch die abgetöteten emotionalen Triebe und Empfindungen der Freikorpsmänner, ihre verhältnismäßige Kälte und vor allem die insgesamt fehlenden Objektbeziehungen - d. h. fehlende Liebe zu einzelnen Menschen im Gegensatz zur vollen Hingabe an de facto abstrakte Einheiten wie „Deutschland“. Die Werte und Handlungen dieser Männer scheinen vor allem durch folgendes repräsentiert zu sein: Pflichtbewusstsein, Tötungs- und Todesbereitschaft, Verdammung der Lüste und Genüsse sowie Praxis der Körperübungen, die oft im Überdrehen der eigenen Kräfte und kurzfristigen Blackouts gipfelten. Methodisch wurde Klaus Theweleit vorgeworfen, dass Informationen zur Auswahl und Bedeutung der untersuchten Texte im Buch fehlten. Die Auswahl scheint mir hinreichend begründet zu sein, hingegen Informationen zur Bedeutung und Auflagen der Bücher aus dem Freikorps-Umfeld habe ich tatsächlich vermisst. So taucht die Frage auf: Welche Rolle haben die untersuchten Texte zur Entstehungszeit gespielt und wie (und in welchem Umfang) wurden sie rezipiert. Schließlich: Inwiefern repräsentieren die im Buch zitierten bzw. dargestellten Männertypen ihre Epoche? Wie gewalttätig waren die kommunistischen Kämpfer und Aufständischen?

Der Autor kommt zu dem Schluss, dass die Mehrheit der Männer dieser Periode in Europa im gewissen Sinne „psychotisch“ war $^{6}$ und Ähnlichkeiten mit dem psychischen Zustand der sieben untersuchten Personen aus dem Freikorpsumfeld aufwiesen. Das soll sich jedenfalls nicht nur auf Deutsche der damaligen Zeit - wenn auch doch im etwas stärkeren Grade als auf andere Europäer bezogen haben. Der Autor schreibt:

Der Begriff Faschismus bezeichnet in ,Männerphantasien“ (2019) eine inhaltlich breit gefasste Bewegung von soldatischen, extrem nationalistischen und gewaltbereiten Personen. Die von Klaus Theweleit im Einzelnen fokussierten Personen sind: der spätere Auschwitz-Kommandant Rudolf Höß; der spätere KZ-Häftling Martin Niemöller; die Freikorpsführer Hermann Ehrhardt und Gerhard Roßbach; Mitglied der Brigade Ehrhardt Manfred von Killinger; der Reichswehr-General Paul von Lettow-Vorbeck; Schriftsteller Ernst von Salomon, der maßgeblich an der Vorbereitung des Attentats gegen den Außenminister Walter Rathenau beteiligt war.

6 D. h. ihre Individuation (Persönlichkeitsbildung) war unfertig (nach der Definition von Margaret S. Mahler), da sie sich von ihren Eltern nie anerkannt fühlten. Ihre Beziehungen zu anderen Menschen wurden dadurch dauerhaft von Angst bzw. Misstrauen und Gefühlskälte geprägt. 
„Ich vermute, dass im wilhelminischen Deutschland nur sehr wenige Menschen das Glück hatten, einigermaßen zu Ende geboren zu werden ${ }^{7}$ [zur Erklärung siehe Fußnote] (und im übrigen Europa nicht sehr viel mehr).“( Theweleit 2019:718)

Theweleit konzediert, dass zwischen einem „normalen“ und quasi „psychotischen“ Menschen eine dünne Linie verläuft und viele Grenzfälle existieren. Außerdem - wie schon klargeworden sein muss - sei der faschistische Typus des Mannes weder auf bestimmte Nationen noch auf spezifische Zeitepochen beschränkt. Unter bestimmten Bedingungen könne er immer und überall entstehen und gedeihen. Der Autor betrachtet seine Protagonisten als Opfer der Gesellschaft, in die sie hineingeboren wurden. Der psychoanalytischen Herangehensweise gemäß stellt er in der frühkindlichen Entwicklung dieser Männer unbefriedigte emotionale Bedürfnisse fest. ${ }^{8}$ Der bedeutendste Erziehungsvorgang jener Zeit, in der besonders viele Menschen des „psychotischen“ Typus großgezogen wurden, war die Prügel. Die mangelnde Zuwendung zum Kleinkind scheint gegen das Ende des 19. Jahrhunderts fast zur allgemeinen Regel geworden zu sein (vgl. Theweleit 2019:458). Die Deprivierten seien bereits als Säuglinge zur Entfremdung von ihren eigenen Körpern geführt worden. Die Ausscheidungsprozesse ihrer Körperflüssigkeiten seien ihnen als besonders ekelhaft eingeprägt worden, obwohl sie ihnen naturgemäß Lust bzw. Entspannung boten. Die Gefühle der körperlichen Lust sollten bei den Kleinkindern mit Schamgefühlen besetzt werden. Die Voraussetzungen für beiderseitig lustvolle körperliche Begegnungen mit anderen Menschen seien infolge dieser Erziehungsweise zerstört worden. Theweleit betont die damalige körperliche Sauberkeitsbesessenheit und nennt die Hygiene ,eine Art [neue] Frömmigkeit“ (ebd. vgl.: 459).

„In der sehr früh einsetzenden Erziehung des Knaben zum soldatischen Mann (und welche bürgerliche wilhelminische Erziehung wollte das nicht sein und welche sozialdemokratische wollte das auch nicht sein) wird das Auftreten all der beschriebenen Feuchtigkeiten [Körpersäfte] außerhalb der speziell dafür vorgesehenen Situationen und Orte unter strengste Strafe gestellt.“ (ebd.)

Diese frühe Erziehung soll später für Angst- und Ekelgefühle gegenüber der Sexualität bei bürgerlichen Männern verantwortlich geworden sein. Die Erziehung (meist zum Soldatentum als Ideal) erweckte Hass gegenüber Proletarierfrauen, bei denen das sexuelle Verhalten viel freier war:

\begin{abstract}
Diese Flintenweiber waren grausame Furien, wie sie nur der Bolschewismus ersinnen konnte. Wenn sich in dem Herzen eines Rotgardisten im Anblick der Leiden unschuldiger Menschen Mitleid regen mochte, diese Weiber waren vertiert und bar jedes menschlichen Gefühls. (Geschrieben vom Nationalsozialisten E. F. Berendt 1935; zit. nach Theweleit 2019:296).
\end{abstract}

Nach Theweleit (2019) tendierten die späteren faschistischen Männer zu Schmerzensakten statt zur Lust, zum Töten statt zum sexuellen Rausch. Ich sehe mich außerstande, die Richtigkeit von Theweleits Thesen in denjenigen Fällen zu beurteilen, wo es sich um psychologische Ursachen mit Wurzeln in den frühen Kindheitsphasen und deren Wirkungen handelt. Theweleits Untersuchung der faschistischen Männer aus dem Freikorps-Umfeld dokumentiert allerdings ziemlich gut ihre Zwangsvorstellungen sowie ihren Hass gegenüber Andersdenkenden, die mit der Gründung der Weimarer Republik an die Macht gelangt sind oder noch gelangen könnten. In den Schriften dieser Männer werden Begriffe wie: Republik, Sozialdemokratie, Kommunismus, Ehrenlosigkeit, Häme, Verfall, Faulheit, Prostitution und Alkoholismus frei assoziativ verknüpft. Der Schriftsteller Ernst Ottwald behauptete zum Beispiel: [I]m übrigen sind die Begriffe Puff, Kneipe, Verbrecher, Kommunisten bei mir unlösbar und unentwirrbar mit einander verknüpft (zitiert nach Theweleit 2019:80). Der Gegensatz in den Schriften der Freikorpsmänner, der vielleicht am meisten überrascht, ist ihre andächtige Rede von der Keuschheit und Aufopferungsbereitschaft der angebeteten engelhaften „weißen“ Frauen, die ihnen als Ideale vorschwebten, während die gleichen Männer kein Erbarmen

Nach Theweleit (2019) sei „das zu Ende Geboren zu werden“ die Voraussetzung dafür, Objektbeziehungen zu bilden, wessen die faschistischen Männer nicht fähig waren und sich dadurch in einem quasi psychotischen Zustand befanden.

$8 \quad$ Anders als die Psychoanalyse lehnt er aber den Ödipus-Komplex als Ursache (nicht nur) bei diesen Männern ab. 
gegenüber den so genannten kommunistischen Flintenweibern zeigten. Im folgenden Teil werde ich den Familienkonflikt im Haushalt der Familie Haueisen in Georg K. Glasers Roman ,Geheimnis und Gewalt' untersuchen, der mit dem oben gesagten in Zusammenhang gebracht werden kann.

\section{Die Spaltung der Familie im Roman ,Geheimnis und Gewalt ${ }^{\boldsymbol{}}$}

Der autodiegetische Erzähler Valentin Haueisen hatte in seiner Kindheit eine sehr liebevolle Beziehung zu seiner Mutter. In ihrem Heimatdorf habe er die einzigen glücklichen Jahre seiner frühen Kindheit erlebt, während der Vater noch im Krieg war. Mehrere Absätze des ersten Kapitels erwecken den Eindruck einer wahren Liebeserklärung an jene Frau, die durch „Liebe, Geduld und Sanftmut" (Glaser 1989:11) ihre Kinder vor der Grausamkeit des Ehemanns zu schützen bestrebt war. Und was noch wichtiger ist: Sie habe Valentins Seele gerettet:

Ich weiß es ganz genau, ich wäre ein Ungeheuer geworden, hätten ihre Arme, mit denen sie die Spannriemen des Alten auffing, sooft sie es konnte, um danach tagelang gramvoll aber ohne Scham blaue Streifen über dem Handgelenk zu tragen, hätten diese heiligen Arme nicht meine Seele gerettet. (ebd.)

Die Prügel des Vaters waren exzessiv, aber trotzdem durchdacht: Er schlug selten hart in aufbrausendem Zorne (ebd.:10). Seine Prügelrituale waren folgendermaßen inszeniert: Er führte seinen noch präpubertären Sohn in die Waschküche (eigentlich einen Keller), wobei er ,seltsam beherrscht blieb“" (ebd.) und die Tür hinter sich sorgfältig schloss. Zum Schlagen benutzte er entweder einen Stock oder meistens einen Spannriemen mit einer großen eisernen Schnalle, die blutende Wunden hinterließ. Wegen nichtiger Verschuldungen schlug er auf den Sohn einfach los. Valentin wollte mutig bleiben, aber schon der Abstieg in den Keller war ihm einfach zu viel (vgl. ebd.):

Ich schrie nach den ersten Hieben um Hilfe, flehte und suchte nach einem Loch in der Wand. Ich näßte und beschmutzte meine Kleider. Und darüber begannen seine Züge [= des Vaters] sich zu verzerren. Er geiferte vor rasender Wut und seine Zähne bleckten fast wollüstig. [...] Er schlug, als wehrte er sich in einem Kampf auf Leben und Tod. (ebd.)

Der Erzähler bietet die Innenperspektive des Vaters nur relativ selten, aber trotzdem wird dem Leser nicht nur das Bild eines unmotiviert schlagwütigen Monsters geliefert. Der Vater bleibt zwar der Todfeind (ebd.:12), trotzdem wird dieser Mann, der sieben Jahre lang [sic] unter Waffen war (ebd.:308) im Roman ebenfalls als ein Mensch seiner Zeit dargestellt, der sich unter spezifischen Bedingungen zu entwickeln hatte. Im vorigen Kapitel habe ich geschrieben, dass die Moral der wilhelminischen Zeit vom Kult der Sauberkeit, Hygiene und Selbstbeherrschung geprägt war. Das Nässen und Beschmutzen (vor Angst) galt infolge dessen innerhalb von soldatischen und bürgerlichen Schichten in Deutschland um 1918 als besonders verabscheuenswürdig. Im zwölften Kapitel von ,Geheimnis und Gewalt' lesen wir, dass Valentins brutaler Vater selbstsicher behauptete, zum Besten seines Kindes zu handeln. Merkwürdig war, daß es ihm blutiger Ernst damit war. Er war sehr ehrlich, verstieß nie gegen die Gesetze (ebd.:305). Aus dem Ersten Weltkrieg brachte er gewisse Erfahrungen und Wertvorstellungen mit, die er beherzigt hatte und zu denen jedenfalls Hierarchie und Gewalt gehörten. Das zeigt sich unter anderem an der Tatsache, dass er noch vor Hitlers Regierungsantritt den Nationalsozialisten beitrat. Der Erzähler von ,Geheimnis und Gewalt ${ }^{\star}$ empfindet, dass er seinen schlagwütigen Vater durch einen stetigen Widerstand provozierte, der in allerkleinsten Verstößen gegen die bürgerliche Kleidungsweise oder in kurzzeitigen Ablenkungen von ständiger Pflichterfüllung bestand. Ich spürte, daß der mörderische Haß des Alten durch meinen Widerstand verursacht war, aber ich konnte mich nicht unterwerfen, denn es ging um mehr als um mein Leben. (ebd.:9f.). Die Hierarchie durfte aus der Sicht des Vaters nicht gestört werden. Aus einem in diesem Kapitel oben angeführten Zitat geht außerdem hervor, dass die mörderischste Wut des Mannes gegenüber seinem Sohn sich gerade in solchen Momenten noch steigerte, wo dieser die Kontrolle über seine Angst verlor und den inzwischen rasend gewordenen Vater bei der Prügel flehend um Nachsicht bat: 
„Lieber Papa, lieber Papa!“ Er aber stieß mich von sich, um den günstigen Abstand zum Schlagen wiederherzustellen. Es kam vor, daß er seinen Spannriemen beiseite warf und mit geballten Fäusten auf mich einschlug, und in solchen Momenten blitzte die Mordlust in seinen Augen. (ebd.: 11)

In dieser Situation bleibt die Hierarchie zwar bestehen, aber der Sohn „versagt“ hier vollständig, wenn der Vater ihn nach den Maßstäben der wilhelminischen und vor allem der Kriegsmoral beurteilt. Folgt der schlagende Mann in solchen Momenten aber noch irgendwelchen Grundsätzen oder unterliegt er dem puren Sadismus? Vielleicht ist in diesem Fall beides präsent. Im ersten Kapitel lesen wir aber, dass der Vater angeblich das Beste für seinen Jungen wünschte. Auch er sei hart angefasst worden (ebd.:19), bekannte er einmal im Anfall von Schwäche und Scham, nachdem Valentin von zu Hause geflüchtet war und zurückgebracht wurde. Man muss durch eine harte Schule gehen, um etwas zu taugen ... denke an deine Zukunft (ebd.), schloss er seine Rede an den Sohn, den er nach diesem Vorfall ausnahmsweise nicht geschlagen hat (vgl. ebd.). Angesichts der wilhelminischen soldatischen Moral hatte der Vater Recht. In Theweleits ,Männerphantasien“ lesen wir interessante Berichte über die Kadettenerziehung in Militäranstalten. Diese habe besonders in der Hierarchiehörigkeit, in der Gewöhnung an körperliche Schmerzen und schließlich in der Bildung einer IchIdentität durch Schläge geführt. Der Kadett sollte dazu geführt werden, Schläge von Tüchtigeren zu akzeptieren und Schläge an Schwächere lustvoll zu verteilen. Bei unsoldatischen Männern führte diese Erziehung zum Schrecken. Im Krieg zeitigte sie aber Erfolg, denn sie hatte die Soldaten von Hemmungen gegenüber dem Feinde befreit. Insofern ist es nicht abwegig zu denken, dass diese Art Erziehung aus der Perspektive des Vaters dem Sohn und dessen Zukunft förderlich sein sollte. Vielleicht bestand das Motiv des Vaters aber noch viel stärker darin, Valentin zur vollständigen und bedingungslosen Unterwerfung unter seine Autorität zu zwingen. Als Soldat oder vielleicht schon als Kind musste der Vater die gleichen Methoden akzeptieren. Es ist ersichtlich geworden, dass dieser Mann die Kriegsmentalität auf den Friedensalltag übertragen hat. Insofern hat er seinem Sohn die Hierarchiehörigkeit und die Gewöhnung an Gewalt und Schmerzen einprägen wollen.

Er war voller Ehrgeiz und der Respekt und das Ansehen der anderen bedeutete ihm alles. Er war sieben Jahre lang [sic] unter Waffen gewesen, und indem dunkle Mächte den Kaiser zu Fall gebracht, hatten sie dessen Soldaten um den einzigen Lohn jener sieben Jahre gebracht: die Ehre war vom Spott besudelt. (ebd.:308)

Valentins Vater war gegen nichts so empfindlich als selbst gegen die entfernte Gefahr, lächerlich zu werden [...] Er wollte todernste Achtung. (ebd.). Seine Verachtung - und zugleich Angst - galt allen, die seiner Meinung nach die Male der Minderwertigkeit und Tücke trugen: Linkshänder, Schielende, Rothaarige, Verwachsene, Juden und Träumer (ebd.) Ebenso hasste er alle Nichtstuer, Neider und Gescheiterte (ebd.:306). Diesen Charakterzug, der von einer krankhaften Angst vor dem Machtverlust zeugt, beobachtet der Erzähler später ebenfalls bei seinem anderen Todfeind, dem Nationalsozialisten Althaus, dessen Brutalität ihre Wurzeln in der panischen Angst vor dem Verlust seiner Kontrolle und Überlegenheit über die anderen hat. Der Fehlschlag der Erziehungsmethoden von Valentins Vater, der später in immer häufigeren Flüchten des Sohnes von zu Hause und schließlich in seinem Anschluss an die Kommunisten den Ausdruck fand, muss dem Mann eine herbe Enttäuschung zugefügt haben. Die Kriegsmentalität hat den Vater sofort nach dessen Rückkehr aus dem Krieg dem Rest der Familie entfremdet. Bereits an den ersten Tagen beobachtet der Erzähler das grobe und besitzergreifende Benehmen des Mannes, den er von nun an „Vater“ nennen soll.

Eines Tages rollten die Kanonen müde und geschlagen aus dem Krieg heim. Einer der zurückkehrenden Soldaten kam bärtig und schmutzig in die Wohnung. Die Mutter schrie auf, umarmte ihn, lachte und weinte zugleich. [...] Der Soldat hing, ohne zu fragen, seinen Rock über die Stuhllehne, setzte sich, schamlos ächzend vor Wohlbehagen, und zog, mit den gleichen rohen Bewegungen gewaltsamer Besitzergreifung, einen Teller an sich. Sooft er sich rührte, entstand eine neue dunkle Farbe hinter meinen Augen, atemraubender und beklemmender als alle bekannten Tönungen. (ebd.:302f.) 
Doch nicht nur die Familie, sondern auch der Vater selbst empfindet eine bittere Enttäuschung über seine Rückkehr zu Hause:

Er [= der Vater] war kaum sechsunddreißig Jahre alt, voller Spannkraft, und hatte aus den fünf Jahren [sic] im Schlamm der Schützengräben einen heißen Lebenshunger aufgespart. Und jetzt, ohne Übergang, jagten ihn hungrige Kinderaugen, eine Frau, die mit dem Fluch der Fruchtbarkeit beladen war und sich so ausschließlich und mächtig den Kindern zugewandt hatte, daß es peinlich war, sie als Geliebte zu denken. (ebd.: 304)

Valentins Vater schalt seine Frau, die scheinbar nur für die Kinder da war ,eine alte Glucke “ (ebd.: 11). Die Frau kümmerte sich voller Sorge um den Haushalt, die Kinder und versuchte mit ihrem dazuverdienten Gelde den Alten zu besänftigen und uns dadurch Schläge zu ersparen (ebd.). Drei von den acht Kindern des Ehepaars sind im Kindesalter gestorben, zwei emigriert, die restlichen zu entweder verschüchterten Wesen oder verstockte[n] blasse[n] Streber[n] geworden. (ebd.: 9). ,Geheimnis und Gewalt' beschreibt den Lebensweg Valentin Haueisens bis zum Ende des Zweiten Weltkriegs, wo er - inzwischen französischer Soldat geworden - aus der deutschen Kriegsgefangenschaft befreit worden ist. Dem kleinbürgerlichen obrigkeitshörigen nationalsozialistischen Vater zum Trotz wurde die Hauptfigur zum Kommunisten - einem idealistischen Träumer von zwischenmenschlicher Solidarität, der jedoch auch in den Strukturen der illegalen Partei noch vor dem Ende des Zweiten Weltkriegs zum Zeugen der Unterdrückung und Machtarroganz wurde. Anders als viele seine Parteigenossen hat er sich der Partei völlig entfremdet. Was die Glaser-Forschung angeht, hat sie in erster Linie gerade den Aspekt der schmerzlichen Beziehung des Autors zur KPD und zum Kommunismus im Allgemeinen untersucht, des Weiteren die Problematik der Emigration und nicht zuletzt das vom späten Glaser beliebte Thema der unabhängigen Gewerbearbeit im Gegensatz zur geisteszermürbenden Massen- (besonders Fließband-) Produktion.

\section{Fazit}

Das Ziel dieser Untersuchung war es, die Familienkonstellation in Georg K. Glasers ,Geheimnis und Gewalt' $z u$ analysieren und vor allem die Spaltung der Familie Haueisen im Roman zu erklären. $\mathrm{Zu}$ diesem Zweck wurden im zweiten und dritten Kapitel einige historische Tatsachen und Theorien präsentiert, die zum besseren Verständnis der Romanhandlung dienen sollten. Obwohl Glasers Roman fiktional ist, basiert er doch auf der Biographie des Autors und - wenn auch literarisch verfremdet und historisch nicht ganz zuverlässig - gibt er die geschichtlich bedingte Atmosphäre in Deutschland in der Epoche der Weimarer Republik wieder. Im Vordergrund des Geschehens steht die Figur des gewalttätigen Vaters, der den Zerfall der Familie verursacht hat. Die historischen Kapitel der vorliegenden Studie weisen auf die Zersplitterung der Gesellschaft hin: auf enorme Ungleichheiten der sozialen Lebensstandards und die Radikalisierung der Ideologien. Außerdem haben die Kriegserfahrungen die zurückgekehrten Soldaten oft tiefgreifend verändert. Im Hinblick auf die Fragestellung des Artikels wird im vierten Kapitel viel Aufmerksamkeit den soldatischen Männern gewidmet, die das Bild Deutschlands besonders stark (spätestens seit der Thronbesteigung Wilhelms II.) bis zum Untergang des „Dritten Reiches“ geprägt haben. Die aus dem Ersten Weltkrieg zurückgekehrten Soldaten bzw. deren nationalistisch-konservative Teile einschließlich Valentin Haueisens Vater unterlagen rechtsradikalen Verschwörungstheorien vom Volksverrat durch unpatriotische Kräfte, die sich später an die Spitze der Weimarer Republik gestellt haben sollen. Die Spaltung der Gesellschaft in Deutschland zwischen 1918 und 1933 äußerte sich nicht nur in der Feindschaft zwischen einzelnen Ideologien und Sozialschichten, sondern drang ebenfalls ins private Leben der Familien ein, wo sie sich sehr häufig in der Form des Vater-Sohn-Konflikts äußerte.

In ,Geheimnis und Gewalt' straft der aus dem Ersten Weltkrieg zurückgekehrte Vater seinen noch (prä-)pubertären Sohn mit Mitteln extremer körperlicher Gewalt. Obwohl die Vergehen des Jungen, wegen derer er brutal geschlagen wird, nichtiger Art sind, spürt der machtbesessene Vater bei seinem Sohn einen andauernden Widerstand, der insgeheim ihm - dem Oberhaupt der Familie - und 
seinen Werten gilt. Der aus dem Krieg zurückgekehrte Mann ist ungemein ehrgeizig, aber gleichzeitig sehr hierarchiebewusst und obrigkeitshörig. Er scheint sowohl von der Brutalität des Krieges als auch von den wilhelminischen Werten entschieden geprägt zu sein. Der Rest der Familie - die Frau und ihre zahlreichen Kinder - fühlen sich dem Mann gegenüber entfremdet. Klaus Theweleit, dessen ,Männerphantasien“ ich als das Hauptwerk der Sekundärliteratur für meine Analyse benutzt habe, versucht die geschichtlich bedingte Mentalität von soldatischen Männern zu erklären. Am überzeugendsten und auf Tatsachen gründend, erscheint Theweleits Beschreibung der Erziehung in militärischen Anstalten und Kollektiven, bzw. im Krieg. Diese habe besonders in der Gewöhnung an Hierarchiehörigkeit und körperliche Schmerzen bestanden. Der Soldat sollte darin trainiert werden, Schläge von Tüchtigeren zu akzeptieren und Schläge an Schwächere lustvoll zu verteilen. Im zivilen Leben führte diese Verhaltens- und Erziehungsweise zum Schrecken und zur Entfremdung (vor allem von der eigenen Familie), wie es der Fall in Glasers Roman ist. Im Krieg zeitigte dies aber Erfolg, denn die Soldaten wurden durch eine solche Erziehung von Hemmungen gegenüber dem Feind befreit. In ,Geheimnis und Gewalt ‘ wurden die Gewalttoleranz und die Hierarchiehörigkeit ebenfalls zu den obersten Ansprüchen des Vaters an den heranwachsenden Sohn. Der Vater wird hier zwar insbesondere durch die Kindsperspektive als ein sadistisches Monster dargestellt; allerding versucht der Erzähler sich auch in dessen psychische Einstellung einzufühlen. Somit kann seine grausame Erziehungsweise nicht nur als ein individuell psychisches, sondern ebenfalls als ein zeittypisches Phänomen verstanden werden.

\section{Literaturverzeichnis}

\section{Primärliteratur:}

Glaser, Georg K. (1989): Geheimnis und Gewalt. Ein Bericht. Frankfurt am Main: Stroemfeld / Roter Stern.

GLASER, Georg K. (2007): Schluckebier und andere Erzählungen. Frankfurt am Main: Stroemfeld.

\section{Sekundärliteratur:}

Brieger, Anton (1973): „,In zwölfter Stunde“. Manfred Kyber. Seher und Dichter. Pforzheim.

KyBER, Manfred (1925): Tierschutz und Kultur. Stuttgart / Heilbronn.

RoHRwASSER, Michael (2007): „Nachwort“. In: Georg K. Glaser: Schluckebier und andere Erzählungen. Frankfurt am Main, S. 323-372.

Rohrwasser, Michael (1989): „Nachwort“. In: Georg K. Glaser: Geheimnis und Gewalt. Ein Bericht. Frankfurt am Main, S. 559-565.

Rohrwasser, Michael (1991): „Georg K. Glaser: Die Partei und das Schreiben“. In: Der Stalinismus und die Renegaten. Die Literatur der Exkommunisten. Stuttgart.

RüHLE, Otto (1922): Das proletarische Kind. Eine Monographie. München.

Theweleit, Klaus (2019): Männerphantasien. Berlin. 